\title{
Peripheral fatigue: has another "threshold" bitten the dust?
}

Mark Burnley

Endurance Research Group, School of Sport and Exercise Sciences, University of Kent, Chatham, Kent, UK.

Address for correspondence:

Dr Mark Burnley

School of Sport and Exercise Sciences

University of Kent

The Medway Building

Chatham Maritime

Kent

ME4 4AG

UK

m.burnley@kent.ac.uk 
In pushing the limits of exercise performance, it is obvious that at some point, a given intensity of exercise will no longer be sustainable, and the performer will have to slow down or stop. One theory explaining why this occurs is that exercise is terminated when adjustments distal to the neuromuscular junction reach a critical threshold level. This theory has three important elements. First, high-intensity endurance exercise results in substantial changes in key metabolites, including, but not limited to, inorganic phosphate and $\mathrm{H}^{+}$. Second, these metabolites activated group III and IV afferent fibers located in muscle interstitial space. Third, the inhibitory feedback transmitted by these afferents reduces the net excitatory input onto motor neurons, causing exercise to be terminated, or the pace to be slowed, on the attainment of a certain (thus "critical") peripheral fatigability, irrespective of exercise duration or intensity (see (1) for review).

In their review of these concepts, Thomas et al. (2) argue that such a threshold does not exist, and that the experimental findings published thus far can be explained by the specificity of the adjustments during fatiguing contractions. Several recent studies have shown that fatigability depends on exercise intensity and exercise modality: exercise with a small muscle mass (such as single knee extensions) is associated with greater levels of fatigability at task failure than that with a large muscle mass (such as cycle ergometry). Thomas et al. (2) suggest that these differences can be explained by the influence of other physiological systems — chiefly the cardiovascular and respiratory systems - on mass-specific $\mathrm{O}_{2}$ delivery and thus the capacity to sustain motor unit activity; during whole-body exercise, mass-specific muscle blood flow is reduced, limiting the muscle mass that can be supported aerobically.

The above observations challenge the concept that adjustments distal to the neuromuscular junction are regulated tightly during exercise; namely, a threshold level of fatigability that can vary so widely does not seem to fulfill a regulatory function. Moreover, the body does not have a means of sensing the level of fatigability in the same way in which experimenters quantify it; that is, using the twitch response to supramaximal nerve stimulation. Instead, as originally 
proposed, it is a threshold concentration of metabolites in the interstitial fluid that is sensed, causing that activation of metaboreceptive afferents. The resulting inhibitory feedback reduces (but does not directly control) the net excitatory input onto motor neurons. If fatigability is regulated during exercise, it is difficult to see how it is being regulated tightly. Yet, many studies show remarkably consistent end-task metabolic profiles, particularly during knee extension tasks above the critical torque or power (see (3) for example). An alternative interpretation of the threshold concept is that it reflects the behavior of ensembles of cells, each cell subject to its own regulatory processes, from which an apparent constraint emerges. It is possible that we have assigned a purpose for something that is simply an emergent property of integrative physiology.

Given the above, has the threshold concept bitten the dust? Not quite. The existence of a threshold based on tolerable adjustments distal to the neuromuscular junction is derived from robust experimental data. In this context, although the model proposed by Thomas et al. (2) is plausible, its validity must be tested also. However, such is the integrative nature of the underlying physiology that developing studies to test the model of Thomas et al. (2) and, crucially, to differentiate it from the predictions of the threshold concept (1) is a supreme experimental challenge. Thus, far from biting the dust, the notion of a peripheral fatigability threshold continues to drive exercise scientists to search for deeper truths about the physiology of human performance.

\section{References:}

1. Hureau TJ, Romer LM Amann M. The 'sensory tolerance limit': A hypothetical construct determining exercise performance? Eur J Sports Sci. 2018;18(1):13-24.

2. Thomas K, Goodall S, and Howatson G. Performance fatigability is not regulated to a peripheral critical threshold. Exerc Sport Sci Rev. 2018;in press. 
3. Vanhatalo A, Fulford J, DiMenna F, Jones AM. Influence of hyperoxia on muscle metabolic responses and the power-duration relationship during severe-intensity exercise in humans: $\mathrm{A}^{31} \mathrm{P}$ magnetic resonance spectroscopy study. Exp Physiol. 2010;95(4):528-540. 